\title{
Frequency and Impact of Preadmission Digestive Symptoms on Outcome in Severe COVID-19: A Prospective Observational Cohort Study
}

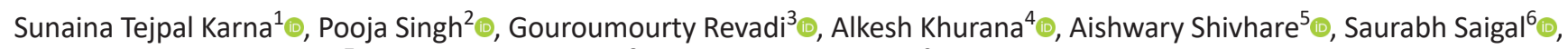
Manoj Kumar Rathiswamy ${ }^{7}{ }^{\circ}$, Jai Prakash Sharma ${ }^{8} \odot$, Vaishali Waindeskar ${ }^{9} \odot$

\begin{abstract}
Background: Coronavirus disease-2019 (COVID-19) commonly presents with respiratory symptoms. However, symptoms involving the digestive system may be present, significance of which is not well studied in the Indian scenario.

Methods: This prospective observational cohort study included consecutive patients with severe COVID-19 admitted to intensive care unit of our tertiary care hospital from September 9, 2020, to March 14, 2021. We evaluated the frequency of preadmission digestive symptoms and compared the demographic, clinical, laboratory parameters, and organ failure at admission and during intensive care along with mortality between those with and without digestive symptoms. In the digestive group, we sought to find predictors of mortality.

Results: Digestive symptoms were present in 76/234 (32.4\%) with severe COVID-19 infection. In comparison to nondigestive group, digestive patients had higher need for noninvasive ventilation ( $p 0.028)$, invasive lines $(68 \%, p 0.003)$, vasopressors $(64 \%, p 0.01)$, blood product transfusion $(21.1 \%, p<0.001)$, and heart failure $(55.4 \%, p 0.041)$. Confounding factors of alcohol abuse, smoking, sedentary lifestyle as a causative agent for heart failure could not be ruled out. Proportional mortality rate is higher in the digestive group $(65.8 \%, p=0.015)$. Mortality is multifactorial with preadmission abdominal pathologies (HR 4.3) or central nervous system (CNS)-related comorbidities (HR 2.829), presentation with multiple digestive symptoms (HR 6.9), higher sequential organ failure assessment score (SOFA) score at admission (HR 1.258) and discharge (HR 1.162), and presence of acute kidney injury (AKI) Grade 3 (HR 2.95) as predictors of mortality. After adjusting for all confounders, need for vasopressor was observed to be associated with 11.58 times higher risk of mortality.

Conclusion: Preadmission digestive symptoms may be associated with a turbulent illness with invasive interventions, heart failure, and greater proportional mortality in severe COVID-19. AKI Grade 3 is identified as a preventable risk factor predicting mortality. CTRI/2021/03/032325.

Keywords: Gastrointestinal complications, Intensive care, Mortality prediction, Severe COVID-19.

Indian Journal of Critical Care Medicine (2021): 10.5005/jp-journals-10071-24020
\end{abstract}

\section{Study Highlights}

In patients with severe coronavirus disease-2019 (COVID-19) needing intensive care, the frequency of preadmission digestive symptoms is $32.47 \%$, with coexistent respiratory symptoms in most. This patient cohort has a more turbulent hospital course with higher invasive interventions, heart failure, and higher proportional mortality. Severe acute kidney injury is a modifiable factor associated with mortality.

\section{INTRODUCTION}

Digestive symptoms have been reported with varied frequency (3-79\%) in coronavirus disease-2019 (COVID-19) pandemic. ${ }^{1,2}$ Preadmission digestive symptoms in COVID-19 patients who need intensive care are not studied in the Indian scenario. Moreover, impact of preadmission digestive symptoms on critical care course of patients with severe COVID-19 is not clear. We hypothesized that the presence of preadmission digestive symptoms in patients with severe COVID-19 may be associated with a more severe course of illness and poorer outcomes as compared to those without digestive symptoms.

With this research gap, we conducted a study to find frequency of preadmission digestive symptoms in severe COVID-19 patients who need intensive care with comparison of course of illness and outcomes between those with and without digestive symptoms.
1,2,5-9 Department of Anesthesiology and Critical Care, All India Institute of Medical Sciences, Bhopal, Madhya Pradesh, India

${ }^{3}$ Department of Community and Family Medicine, All India Institute of Medical Sciences, Bhopal, Madhya Pradesh, India

${ }^{4}$ Department of Pulmonary Medicine, All India Institute of Medical Sciences, Bhopal, Madhya Pradesh, India

Corresponding Author: Sunaina Tejpal Karna, Department of Anesthesiology and Critical Care, All India Institute of Medical Sciences, Bhopal, Madhya Pradesh, India, Phone: +91 9540946869, e-mail: drtejpal@gmail.com

How to cite this article: Karna ST, Singh P, Revadi G, Khurana A, Shivhare A, Saigal $S$, et al. Frequency and Impact of Preadmission Digestive Symptoms on Outcome in Severe COVID-19: A Prospective Observational Cohort Study. Indian J Crit Care Med 2021;25(11): 1247-1257.

Source of support: Nil

Conflict of interest: None

\section{Materials and Methods Study Design and Subjects}

This prospective cohort study was conducted in an academic Institute in Central India after institutional ethical committee

(c) The Author(s). 2021 Open Access This article is distributed under the terms of the Creative Commons Attribution 4.0 International License (https://creativecommons. org/licenses/by-nc/4.0/), which permits unrestricted use, distribution, and non-commercial reproduction in any medium, provided you give appropriate credit to the original author(s) and the source, provide a link to the Creative Commons license, and indicate if changes were made. The Creative Commons Public Domain Dedication waiver (http://creativecommons.org/publicdomain/zero/1.0/) applies to the data made available in this article, unless otherwise stated. 
approval (IHEC-LOP/2020/IM0279) with a waiver of informed consent. We included consecutive patients above 18 years admitted to our intensive care unit (ICU) from September 9, 2020, to March 14, 2021, with confirmed COVID-19 with positive RT-PCR of paired nasopharyngeal and throat swab.

\section{Data Collection}

Clinical course of each patient was followed from admission until discharge or mortality.

- Admission: We noted demographic details, clinical characteristics, vital signs, laboratory investigations, sequential organ failure assessment score (SOFA score) with detailed history within 15 days of COVID-19-positive testing. Digestive system involvement was defined with the presence of at least one symptom out of loss of appetite, nausea, vomiting, diarrhea (loose stools $\geq 3$ per day), abdominal pain, abdominal distension, or gastrointestinal bleeding (hematemesis, melena).

- During course of ICU stay: An independent observer blinded about initial symptoms recorded the medications (antiviral, diuretics, tissue plasminogen activator, vasopressors, and antiarrhythmics), invasive vascular lines, renal replacement therapy (RRT), noninvasive (NIMV) or invasive mechanical ventilation (IMV) during treatment along with vitals, and investigations on the third, fifth, and last day of ICU. We noted SOFA score on the last day of ICU stay. New organ injury during the treatment was recorded, including shock, arrhythmias, left ventricular failure, neurological complications, thromboembolic complications, acute respiratory distress syndrome (ARDS) by Berlin definition, acute kidney injury (AKI) by acute kidney injury network (AKIN), and acute liver injury (ALI) [alanine aminotransferase (ALT) more than $50 \mathrm{lU} / \mathrm{mL}$, with 5, 10, >10 times rise graded as mild, moderate, and severe, respectively.

- Final outcome: We noted the mortality, duration from symptom onset to hospital admission, discharge, or death along with the total length of ICU and hospital stay.

\section{Statistical Analysis}

Data were entered, cleaned, and coded in Microsoft excel 2013. Data analysis was done using IBM SPSS version 24. Continuous variables were summarized as mean (SD) when normally distributed and as median (IQR) when nonnormally distributed. Chi-square and Fischer exact tests were used for testing the association between categorical variables. We used unpaired $t$-test/Mann-Whitney for continuous variables based on distribution. Factors predicting the digestive symptom at presentation were analyzed using multivariable logistic regression.

For the purpose of analysis, depending on the presence or absence of digestive symptoms, we categorized the cohort into either digestive or nondigestive groups, respectively. Cox proportional regression analysis was run with time to event as the duration of days from ICU admission to death and event as mortality, and the significant predictors of mortality were outlined. Multivariate Cox regression was applied among the significant predictors from univariable Cox analysis. Among the laboratory parameters and severity scores which turned significant, their respective cutoff parameters were determined using receiver- operating characteristic (ROC) curve, with area under the curve more than $50 \%$ and $p$ value $<0.05$ as significant.

\section{Results \\ Patients Flow and Baseline Characteristics}

A total of 1,338 symptomatic patients were admitted to our tertiary care institute with COVID-19 infection during the study period, of which 234 patients with severe COVID-19 infection who needed intensive care were enrolled in our study. The baseline characteristics of these patients are provided in Table 1. The details of the comorbidities and systemic presentations are outlined in Supplementary Table 1. The median (IQR) duration from symptom onset to ICU admission was 5 (3-7) days. ALI was observed in 72 (30.8\%) patients with $62(26.5 \%), 8(3.4 \%)$, and $2(0.9 \%)$ patients presenting with mild, moderate, and severe ALI, respectively. Median SOFA score at admission was 4 (IQR 3-5).

\section{Frequency and Clinical Spectrum of Digestive Symptoms}

Digestive symptoms were present in 76/234 (32.4\%) and absent in $158 / 234$ patients (67.5\%) with severe COVID-19 infection admitted to ICU. Only 4/234 (1.7\%) patients were presented exclusively with digestive symptoms. The clinical spectrum of digestive symptoms is given in Figure 1. Diarrhea was usually not clinically severe.

\section{Characteristics of Patients with Digestive Symptoms}

The average age of the patients with digestive symptoms was $59( \pm 12)$ years and the male to female ratio was 1.8:1. Thirty-one (45.6\%) patients were overweight, followed by normal, obese, and underweight categories (Table 1). Comorbidities were present in $67(88.2 \%)$ patients and have been listed in Supplementary Table 1. Dyspnea, fever, and cough were the most common nondigestive symptoms in this group (Table 1).

On application of multivariate regression model to identify predictors of digestive symptoms at admission, males had 3.337 times higher odds of presenting with digestive symptoms. Both low body mass index (BMI) (aOR 0.85, $p$ 0.019) and fibrinogen levels (aOR 0.997, $p$ 0.034) were more associated with gastrointestinal involvement (Table 2).

\section{Comparison of Digestive and Nondigestive Groups Using Univariate Analysis}

\section{At Admission}

The univariable analysis of demographic, clinical, laboratory parameters at admission is listed in Table 1. Patients with digestive symptoms had a lower diastolic blood pressure $(p=0.037)$ (Table 1). Fever, cough, and shortness of breath were more common in nondigestive group (Supplementary Table 1). Hepatobiliary diseases were exclusively present in the digestive group ( $p=0.004$ ). The SOFA score was comparable between digestive and nondigestive groups ( $p=0.517$ ).

At admission, the digestive group had higher lactate and alkaline phosphatase ( $p$ values 0.01 and 0.034 , respectively) and lower platelet and fibrinogen values ( $p$ values 0.005 and 0.038 , respectively). C-reactive protein (CRP) though higher in the digestive group (139.5 vs 93.5) did not achieve statistical significance $(p=0.065)$. 
Table 1: Univariable analysis of clinical, laboratory parameters, and outcomes

\begin{tabular}{|c|c|c|c|c|}
\hline & Total & $\begin{array}{l}\text { Digestive symptoms present } \\
\qquad N=76\end{array}$ & $\begin{array}{l}\text { Digestive symptoms absent } \\
\qquad N=158\end{array}$ & $p$ value \\
\hline Age mean (SD) & $57.7(13.25)$ & $59(12)$ & $57(14)$ & 0.218 \\
\hline \multicolumn{5}{|l|}{ Gender $n(\%)$} \\
\hline Male & $162(69.2)$ & $49(64.5)$ & $113(71.5)$ & 0.274 \\
\hline Female & $72(30.8)$ & $27(35.5)$ & $45(28.5)$ & \\
\hline \multicolumn{5}{|l|}{ BMI $n(\%)$} \\
\hline$<18.5$ & $12(5.1)$ & $7(10)$ & $5(4)$ & 0.148 \\
\hline $18.5-22.9$ & $42(17.9)$ & $18(26.5)$ & $24(19)$ & \\
\hline $23-27.4$ & $97(41.5)$ & $31(45.6)$ & $66(52.4)$ & \\
\hline$>27.5$ & $43(18.4)$ & $12(17.6)$ & $31(24.6)$ & \\
\hline \multicolumn{5}{|l|}{ Comorbidities } \\
\hline No & $38(16.2)$ & $9(11.1)$ & $29(18.4)$ & 0.206 \\
\hline Yes & $196(83.8)$ & $67(88.2)$ & $121(81.6)$ & \\
\hline \multicolumn{5}{|l|}{ Parameters at admission } \\
\hline \multicolumn{5}{|l|}{ Vitals-mean (SD) } \\
\hline PR & $91.4(19.6)$ & $92(19)$ & $91(20)$ & 0.802 \\
\hline SBP & $131.4(20.6)$ & $129(21)$ & $133(21)$ & 0.154 \\
\hline DBP & $78.7(13.4)$ & $76(14)$ & $80(13)$ & 0.037 \\
\hline $\mathrm{RR}$ & $29.4(7.4)$ & $30(8)$ & $29(7)$ & 0.764 \\
\hline SOFA median (IQR) & $4(3-5)$ & $3(3-5)$ & $4(3-4)$ & 0.517 \\
\hline \multicolumn{5}{|l|}{ Blood investigations } \\
\hline \multicolumn{5}{|l|}{ CBC mean (SD) } \\
\hline Hemoglobin & $12.4(2.31)$ & $12(2.7)$ & $12.6(2.1)$ & 0.108 \\
\hline Total leukocyte count & $11.3(5.53)$ & $10.9(5.5)$ & $11.45(5.56)$ & 0.481 \\
\hline Platelets & $236(105.1)$ & $207.9(108.3)$ & $249.46(101.1)$ & 0.005 \\
\hline \multicolumn{5}{|l|}{ Coagulation tests mean (SD) } \\
\hline INR & $1.2(0.47)$ & $1.3(0.5)$ & $1.2(0.5)$ & 0.344 \\
\hline Fibrinogen & $566.5(203.6)$ & $514(172)$ & $593(214)$ & 0.038 \\
\hline aPTT & $32.54(13.01)$ & $31.6(9)$ & $33(14.6)$ & 0.512 \\
\hline \multicolumn{5}{|l|}{ Cytokines test median (IQR) } \\
\hline D-dimer & $1.02(0.5-1.8)$ & $1.1(0.6-1.5)$ & $0.9(0.5-1.9)$ & 0.937 \\
\hline C-reactive protein & $107.5(58.17-194.5)$ & $139.5(67-200)$ & $93.5(53-172)$ & 0.065 \\
\hline Neutrophil-lymphocyte ratio & $11(6.8-18.8)$ & $11(6.8-18.2)$ & $11(7-18.8)$ & 0.817 \\
\hline \multicolumn{5}{|l|}{ Liver function tests median (IQR) } \\
\hline Total bilirubin & $0.65(0.5-0.9)$ & $0.7(0.5-1)$ & $0.7(0.5-0.9)$ & 0.93 \\
\hline Direct bilirubin & $0.2(0.12-0.3)$ & $0.2(0.1-0.4)$ & $0.2(0.1-0.3)$ & 0.227 \\
\hline Aspartate aminotransferase & $44.85(29-67.75)$ & $45(31-66)$ & $43(29-68)$ & 0.477 \\
\hline Alanine aminotransferase & $34(23-55)$ & $34(23-56)$ & $34(23-54)$ & 0.933 \\
\hline Alkaline phosphatase & $90.5(69-123.25)$ & $107(73-140)$ & $88(68-119)$ & 0.034 \\
\hline Albumin & $3.17(2.89-3.4)$ & $3.1(2.8-3.4)$ & $3.2(2.9-3.4)$ & 0.34 \\
\hline Lactate & $1.6(0.93)$ & $1.9(1)$ & $1.5(0.9)$ & 0.01 \\
\hline \multicolumn{5}{|l|}{ Kidney function tests } \\
\hline Urea median (IQR) & $46(29-71)$ & $46(33-78)$ & $46.5(28-68)$ & 0.518 \\
\hline Creatinine median (IQR) & $0.99(0.80-1.29)$ & $1(0.8-1.5)$ & $1(0.8-1.2)$ & 0.598 \\
\hline Sodium mean (SD) & $134(5.28)$ & $134(5)$ & $135(5)$ & 0.51 \\
\hline Potassium mean (SD) & $4.2(0.73)$ & $4.3(0.7)$ & $4.2(0.7)$ & 0.712 \\
\hline \multicolumn{5}{|c|}{ Organ-specific complications at admission } \\
\hline Acute liver injury & $72(30.8)$ & $24(31.6)$ & $48(30.4)$ & 0.852 \\
\hline \multicolumn{5}{|l|}{ Severity of acute liver injury } \\
\hline Mild & $62(26.5)$ & $20(26.3)$ & $42(26.6)$ & 0.944 \\
\hline
\end{tabular}


Table 1: (Contd...)

\begin{tabular}{|c|c|c|c|c|}
\hline & Total & $\begin{array}{l}\text { Digestive symptoms present } \\
\qquad N=76\end{array}$ & $\begin{array}{l}\text { Digestive symptoms absent } \\
\qquad N=158\end{array}$ & $p$ value \\
\hline Moderate & $8(3.4)$ & $3(3.9)$ & $5(3.2)$ & \\
\hline Severe & $2(0.9)$ & $1(1.3)$ & $1(0.6)$ & \\
\hline \multicolumn{5}{|l|}{ ARDS } \\
\hline Mild & $53(22.6)$ & $19(25)$ & $34(21.5)$ & 0.747 \\
\hline Moderate & $106(45.3)$ & $35(46)$ & $71(44.9)$ & \\
\hline Severe & $62(26.5)$ & $17(22.4)$ & $45(28.5)$ & \\
\hline Absent & $13(5.6)$ & $5(6.6)$ & $8(5.1)$ & \\
\hline \multicolumn{5}{|c|}{ Interventions and therapy during treatment } \\
\hline Need of antivirals & $101(43.2)$ & $30(73.2)$ & $71(75.5)$ & 0.771 \\
\hline $\begin{array}{l}\text { Median (IQR) days of oxygen } \\
\text { supplementation }\end{array}$ & $7.5(5-13)$ & $8(5-15)$ & $7(5-12)$ & 0.454 \\
\hline Noninvasive ventilation & $206(88)$ & $72(94.7)$ & $134(84.8)$ & 0.028 \\
\hline $\begin{array}{l}\text { Duration of noninvasive ventilation } \\
\text { median (IQR) days }\end{array}$ & $4(2-6)$ & $5(3-7)$ & $4(2-6)$ & 0.160 \\
\hline Invasive mechanical ventilation & 131 (55.98\%) & $35(46.1 \%)$ & $96(60.8 \%)$ & 0.034 \\
\hline $\begin{array}{l}\text { Median (IQR) days of invasive } \\
\text { ventilation }\end{array}$ & $5(3-9)$ & $5(3-8)$ & $6(3-9)$ & 0.725 \\
\hline Invasive line & $125(53.4)$ & $51(68)$ & $74(47.1)$ & 0.003 \\
\hline Diuretics & $71(30.3)$ & $23(30.3)$ & $48(30.4)$ & 0.868 \\
\hline Renal replacement therapy & $20(8.5)$ & $8(10.7)$ & $12(7.6)$ & 0.443 \\
\hline Tissue plasminogen activator & $28(12)$ & $8(14.8)$ & $20(16.4)$ & 0.792 \\
\hline Need of transfusion $n(\%)$ & $24(10.3)$ & $16(21.1)$ & $8(5.1)$ & $<0.001$ \\
\hline Surgery & $10(4.3)$ & $3(3.9)$ & $7(4.4)$ & 0.864 \\
\hline \multicolumn{5}{|c|}{ Complications during the course of hospital stay } \\
\hline Acute kidney injury & $105(44.9)$ & $39(51.3)$ & $66(41.8)$ & 0.169 \\
\hline \multicolumn{5}{|l|}{$\begin{array}{l}\text { Acute kidney injury network severity } \\
\text { grade }(n=105)\end{array}$} \\
\hline 1 & $67(28.6)$ & $27(35.5)$ & $40(25.3)$ & 0.429 \\
\hline 2 & $15(6.4)$ & $5(6.6)$ & $10(6.3)$ & \\
\hline 3 & $23(9.8)$ & $7(9.2)$ & $16(10.1)$ & \\
\hline CNS complications & $14(6)$ & $4(5.3)$ & $10(96.3)$ & 0.747 \\
\hline Thromboembolic complications & $27(11.5)$ & $8(10.8)$ & $19(12)$ & 0.788 \\
\hline \multicolumn{5}{|l|}{ Acute liver injury } \\
\hline Aspartate aminotransferase $>50$ & $96(41)$ & $34(44.7)$ & $62(39.2)$ & 0.423 \\
\hline Alanine aminotransferase $>50$ & $68(29.1)$ & $23(30.3)$ & $45(28.5)$ & 0.779 \\
\hline Heart failure & $105(44.9)$ & $41(55.4)$ & $64(41)$ & 0.041 \\
\hline Arrhythmias $n(\%)$ & 49 (20.9) & $16(21.3)$ & $33(20.9)$ & 0.938 \\
\hline Shock & $118(50.4)$ & $37(48.7)$ & $81(51.3)$ & 0.711 \\
\hline \multicolumn{5}{|l|}{ ARDS at last ICU day } \\
\hline Mild & $31(13.2)$ & $5(6.6)$ & $26(16.5)$ & 0.103 \\
\hline Moderate & $60(25.6)$ & $17(22)$ & $43(27.2)$ & \\
\hline Severe & $50(21.4)$ & $18(23.7)$ & $32(20.3)$ & \\
\hline SOFA score at ICU discharge & $5(2-8)$ & $6(2-9)$ & $4(2-8)$ & 0.051 \\
\hline \multicolumn{5}{|l|}{ Outcome } \\
\hline Mortality & $128(54.7)$ & $50(65.8)$ & $78(49.4)$ & 0.015 \\
\hline \multicolumn{5}{|c|}{ Time-based outcomes in hospitalization } \\
\hline Total ICU stay & $8(5-14)$ & $8(5-16)$ & $8(5-13)$ & 0.291 \\
\hline Total hospital stay & $12(7-18)$ & $13(6.5-18.5)$ & $11(8-18)$ & 0.893 \\
\hline Symptom onset to ICU admission & $5(3-7)$ & $5(3-8)$ & $4(3-7)$ & 0.064 \\
\hline ICU admission to death & $3.5(0-10)$ & $5(0-10)$ & $1(0-9)$ & 0.179 \\
\hline ICU admission to hospital discharge & $0(0-10)$ & $0(0-8)$ & $1(0-10)$ & 0.876 \\
\hline Symptom onset to discharge/death & $15(11-21)$ & $16(11-24)$ & $15(11-21)$ & 0.296 \\
\hline
\end{tabular}




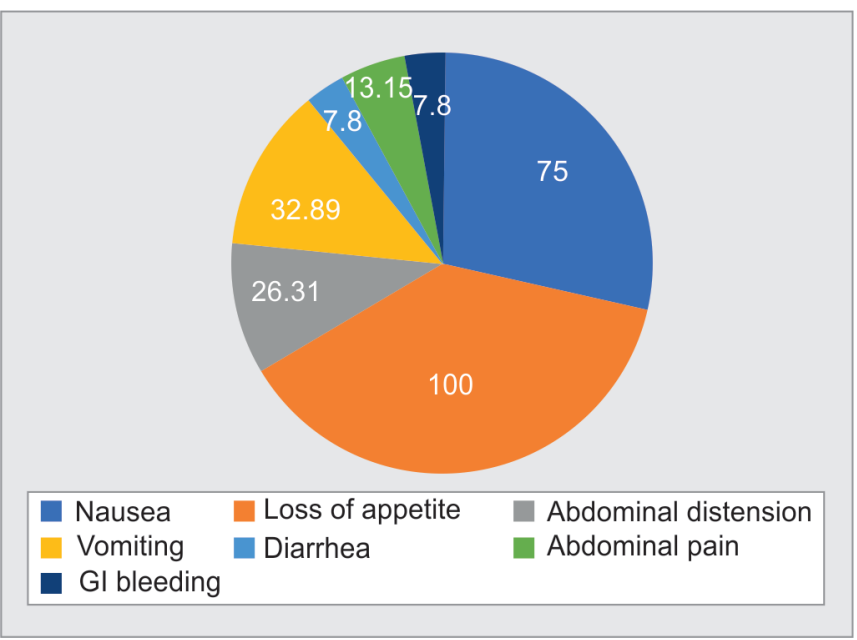

Fig. 1: Digestive symptoms distribution as $n(\%)$

Table 2: Multivariate logistic regression model for identifying predictors of digestive symptom at the time of admission

\begin{tabular}{lccc}
\hline Variables & $\begin{array}{c}\text { Adjustable } \\
\text { odd's ratio }\end{array}$ & $\begin{array}{c}\text { p value } \\
\text { Age confidence } \\
\text { interval }\end{array}$ \\
\hline $\begin{array}{l}\text { Male } \\
\text { Female (Ref) }\end{array}$ & 1.012 & 0.428 & $0.977-1.057$ \\
BMl & 3.337 & 0.050 & $0.999-11.153$ \\
Presence of comorbidity & 0.850 & 0.019 & $0.741-0.973$ \\
No (ref) & - & - & - \\
1 comorbidity & 2.393 & 0.374 & $0.349-16.4$ \\
$\geq 2$ comorbidity & 1.218 & 0.829 & $0.204-7.272$ \\
At the time of admission & & & \\
Platelets & 0.998 & 0.583 & $0.993-1.004$ \\
Total bilirubin & 1.235 & 0.593 & $0.569-2.682$ \\
Aspartate aminotransferase & 0.984 & 0.148 & $0.964-1.006$ \\
Alanine aminotransferase & 1.013 & 0.280 & $0.990-1.036$ \\
C-reactive protein & 1.003 & 0.534 & $0.998-1.008$ \\
Alkaline phosphatase & 1.010 & 0.119 & $0.997-1.022$ \\
Fibrinogen & 0.997 & 0.034 & $0.995-1.000$ \\
\hline
\end{tabular}

\section{Course of Hospital Stay}

There was higher need for NIMV in the digestive group $(p=0.028)$ with median duration of 5 days (IQR 3-7). The need for invasive lines (68 vs $47 \%, p=0.003$ ) and vasopressors (64 vs $45.8 \%, p=0.01$ ) was more in the digestive group. Nondigestive group had higher need for IMV as expected. Blood product transfusion was higher in the digestive group ( 21.1 vs $5.1 \%, p<0.001)$. More patients with digestive symptoms developed heart failure ( 55.4 vs $41 \%, p=0.041$ ). The SOFA score was still statistically higher at ICU discharge with a median value of 6 (IQR 2-9) in digestive group (Table 1).

Supplementary Table 2 provides the changes in the vital signs and laboratory parameters on admission, third, fifth, and last days of ICU. Association was not applied as multiple confounders were present. Digestive group had a higher fall in hemoglobin and albumin levels and a rise in total leukocyte count, neutrophillymphocyte ratio (NLR), AST, and ALT during illness. The mean $\mathrm{PaO}_{2} / \mathrm{FiO}_{2} \mathrm{P} / \mathrm{F}$ ratio was observed to rise in the nondigestive group.

\section{Outcome}

Proportional mortality was higher in digestive as compared to the nondigestive group ( 65.8 vs $49.4 \%, p=0.015$ ). The duration of illness from symptom onset to ICU discharge, ICU and hospital stay was similar in both the groups.

\section{Predictors of Mortality in Digestive Group}

Table 3 describes the results of Cox proportional hazard used for univariate analysis of predictors of mortality among 76 patients with digestive symptoms. Factors associated with raised risk of mortality were the presence of neurological comorbidity (HR $4.3, p=0.02$ ), hepatobiliary disease (HR 2.89, $p=0.055$ ), five preadmission digestive symptoms (HR 6.9, $p=0.046$ ), higher SOFA score at admission (HR 1.2) and discharge (HR 1.16), and Grade 3 AKI (HR 2.9) during the treatment. SOFA score of 5.5 at admission predicted mortality with a sensitivity of $36 \%$ and specificity of $100 \%$ while a SOFA score of 4.5 at discharge predicted mortality with $86 \%$ sensitivity and $100 \%$ specificity (Fig. 2). The increase in duration of NIMV and IMV was protective against mortality in the digestive group along with duration of ICU and hospital stay.

The need for vasopressor use was associated with 11.53 times increased risk of mortality $(p=0.016)$ on Cox proportional hazard multivariate regression analysis run with all the significant variables from univariate regression along with demographic parameters, number of symptoms at presentation, comorbidities, laboratory tests, organ-specific complications at admission, therapeutic strategies, complications during illness, and days of ICU and hospital stay.

\section{Discussion}

To the best of our knowledge, this is the first study from India in patients with severe COVID-19 illness requiring intensive care, which showed the following:

- Digestive symptoms are present in $32.47 \%$ of patients with association with male gender, low BMI, and low fibrinogen.

- Digestive group has higher need for invasive lines, vasopressor use, blood transfusion, and noninvasive ventilation; higher incidence of heart failure; and raised mean SOFA score at last ICU day with a higher proportional mortality.

- Mortality in digestive group was multifactorial in digestive group with preadmission abdominal pathologies or central nervous system (CNS) comorbidities, multiple (five or more) digestive symptoms, higher SOFA score at admission and discharge, and severe AKI during illness. There was no significant association with days of respiratory support (NIMV or IMV). On multivariate analysis, the need for vasopressors had 11.53 times higher risk of mortality.

Digestive symptoms in COVID-19 have been reported earlier in the community-based survey or hospitalized inpatients., ${ }^{1,5}$ An earlier study in North India done on follow-up of initial COVID-19 diagnosis found that among 44 symptomatic patients with COVID-19, 25\% had only gastrointestinal symptoms while combined nongastrointestinal symptom was present in $34.1 \%$ of patients. ${ }^{6}$ Though digestive symptoms may seem trivial, they have been noted to be associated with increased severity of illness. ${ }^{4}$ However, the frequency of digestive symptoms in patients with severe COVID-19 is not reported in an Indian context.

Regional variation in the digestive symptoms in COVID-19 is seen with a higher prevalence in North America followed by 
Table 3: Univariable analysis of predictors of mortality among patients presenting with digestive symptoms: (Cox regression)

\begin{tabular}{|c|c|c|c|c|c|}
\hline & Survived $(n=26)$ & $\operatorname{Died}(n=50)$ & Hazard ratio & p value & $95 \% \mathrm{Cl}$ \\
\hline Age mean (SD) & $57(13.7)$ & $60.3(11.5)$ & 0.989 & 0.438 & $0.96-1.02$ \\
\hline \multicolumn{6}{|l|}{ Gender $n(\%)$} \\
\hline Male & $15(57.7)$ & $34(68)$ & 0.886 & 0.699 & $0.47-1.64$ \\
\hline Female (ref) & $11(42.3)$ & $16(32)$ & - & - & - \\
\hline \multicolumn{6}{|l|}{ Body mass index $n(\%) \#$} \\
\hline$<18.5$ (ref) & $2(8.3)$ & $5(11.4)$ & - & - & - \\
\hline $18.5-22.9$ & $7(29.2)$ & $11(25)$ & 0.874 & 0.809 & $0.29-2.60$ \\
\hline $23-27.4$ & $10(41.7)$ & $21(47.7)$ & 0.525 & 0.208 & $0.19-1.43$ \\
\hline$>27.5$ & $5(20.8)$ & $7(15.9)$ & 0.828 & 0.753 & $0.26-2.68$ \\
\hline \multicolumn{6}{|c|}{ Preadmission digestive symptoms } \\
\hline Only 1 symptom & $3(11.5)$ & $2(4)$ & - & - & - \\
\hline 2 symptoms & $12(46.2)$ & $19(38)$ & 0.955 & 0.950 & $0.22-4.14$ \\
\hline 3 symptoms & $9(34.6)$ & $17(34)$ & 1.180 & 0.827 & $0.27-5.19$ \\
\hline 4 symptoms & $2(7.7)$ & $9(18)$ & 1.092 & 0.911 & $0.23-5.10$ \\
\hline 5 symptoms & 0 & $3(6)$ & 6.953 & 0.046 & $1.03-46.8$ \\
\hline \multicolumn{6}{|l|}{ Comorbidities } \\
\hline No (ref) & $2(7.7)$ & $7(14)$ & - & - & - \\
\hline Yes & $24(92.3)$ & $43(86)$ & 0.469 & 0.075 & $0.20-1.08$ \\
\hline \multicolumn{6}{|l|}{ Type of comorbidity } \\
\hline Diabetes & $13(50)$ & $31(62)$ & 0.734 & 0.298 & $0.41-1.31$ \\
\hline Hypertension & $13(50)$ & $31(62)$ & 0.734 & 0.298 & $0.41-1.31$ \\
\hline Coronary artery disease & $4(15.4)$ & $5(10)$ & 0.491 & 0.179 & $0.17-1.38$ \\
\hline Neurological & $2(7.7)$ & $3(6)$ & 4.310 & 0.020 & $1.26-14.73$ \\
\hline Chronic kidney disease & 0 & $5(10)$ & 1.40 & 0.479 & $0.55-3.56$ \\
\hline Hepatobiliary & 0 & $4(8)$ & 2.829 & 0.055 & $0.97-8.19$ \\
\hline Endocrine & $5(19.2)$ & $5(10)$ & 0.594 & 0.328 & $0.21-1.68$ \\
\hline Respiratory & 0 & $6(12)$ & 1.184 & 0.701 & $0.49-2.18$ \\
\hline Obstructive sleep apnea & 0 & $1(2)$ & 2.86 & 0.310 & $0.37-21.68$ \\
\hline Malignancy & 0 & $2(4)$ & 3.505 & 0.093 & $0.81-15.16$ \\
\hline \multicolumn{6}{|c|}{ Laboratory parameters at admission } \\
\hline Hemoglobin & $12.8(11.3-14)$ & $12.2(10.5-13.5)$ & 0.984 & 0.750 & $0.888-1.089$ \\
\hline Total leukocyte count & $8.96(6.88-11.38)$ & $9.95(7.3-13.5)$ & 0.629 & 1.012 & $0.965-1.061$ \\
\hline Platelets & $218.5(196.5-264)$ & $180(117-239)$ & 0.999 & 0.416 & $0.996-1.002$ \\
\hline Aspartate aminotransferase & $44(26-58)$ & $49(33-84)$ & 1.000 & 0.508 & $0.999-1.001$ \\
\hline Alanine aminotransferase & $28(23-45)$ & $37.3(24-65)$ & 1.000 & 0.477 & $0.999-1.002$ \\
\hline Alkaline phosphatase & $98(72.5-140.5)$ & $108(76-128)$ & 1.000 & 0.580 & $0.998-1.004$ \\
\hline Fibrinogen & $499(351-650)$ & $482(408-584)$ & 1.000 & 0.998 & $0.998-1.002$ \\
\hline C-reactive protein & $111(76-192)$ & $144(67-200)$ & 1.001 & 0.676 & $0.998-1.004$ \\
\hline Lactate & $2(1.2-2.2)$ & $1.8(1.1-2.5)$ & 1.038 & 0.815 & $0.761-1.415$ \\
\hline Creatinine & $0.9(0.8-1)$ & $1.1(0.8-1.5)$ & 1.128 & 0.427 & $0.838-1.517$ \\
\hline \multicolumn{6}{|l|}{ Complications } \\
\hline \multicolumn{6}{|l|}{ Arrhythmias ${ }^{*} n(\%)$} \\
\hline Absent (ref) & $24(96)$ & $35(70)$ & - & - & - \\
\hline
\end{tabular}




\begin{tabular}{|c|c|c|c|c|c|}
\hline Present & $1(4)$ & $15(30)$ & 0.799 & 0.472 & $0.43-1.47$ \\
\hline \multicolumn{6}{|l|}{ Heart failure ${ }^{* *}$} \\
\hline Absent (ref) & $7(28)$ & $26(53.1)$ & - & - & - \\
\hline Present & $18(72)$ & $23(46.9)$ & 0.868 & 0.628 & $0.49-1.54$ \\
\hline \multicolumn{6}{|l|}{ Acute liver injury at admission } \\
\hline Absent (ref) & $21(80.8)$ & $31(62)$ & - & - & - \\
\hline Present & $5(19.2)$ & $19(28)$ & 0.686 & 0.205 & $0.38-1.23$ \\
\hline \multicolumn{6}{|c|}{ Severity of acute liver injury $(n=72)$} \\
\hline Absent (ref) & $21(80.8)$ & $31(62)$ & - & - & - \\
\hline Mild & $5(19.2)$ & $15(30)$ & 0.730 & 0.327 & $0.39-1.37$ \\
\hline Moderate & 0 & $3(6)$ & 0.442 & 0.188 & $0.13-1.49$ \\
\hline Severe & 0 & $1(2)$ & 1.755 & 0.585 & $0.23-13.2$ \\
\hline \multicolumn{6}{|c|}{ Acute respiratory distress syndrome at admission } \\
\hline Absent (ref) & $4(15.4)$ & $1(2)$ & - & - & - \\
\hline Mild & $6(23.1)$ & $13(26)$ & 1.46 & 0.718 & $0.19-11.33$ \\
\hline Moderate & $13(50)$ & $22(44)$ & 0.98 & 0.987 & $0.13-7.38$ \\
\hline Severe & $3(11.5)$ & $14(28)$ & 1.48 & 0.707 & $0.19-11.39$ \\
\hline \multicolumn{6}{|c|}{ Acute respiratory distress syndrome at last day in ICU } \\
\hline Absent (ref) & $2(7.7)$ & $34(68)$ & - & - & - \\
\hline Mild & $4(15.4)$ & $1(2)$ & 1.331 & 0.780 & $0.18-9.92$ \\
\hline Moderate & $14(53.8)$ & $3(6)$ & 2.166 & 0.215 & $0.64-7.35$ \\
\hline Severe & $6(23.1)$ & $12(24)$ & 0.623 & 0.203 & $0.30-1.29$ \\
\hline \multicolumn{6}{|l|}{$\mathrm{AKI}$ during treatment } \\
\hline Absent (ref) & $18(69.2)$ & $19(38)$ & - & - & - \\
\hline Present & $8(30.8)$ & $31(62)$ & 1.838 & 0.052 & $0.99-3.40$ \\
\hline \multicolumn{6}{|l|}{ Severity of AKI } \\
\hline 0 (ref) & $18(69.2)$ & $19(38)$ & - & - & - \\
\hline Grade 1 & $7(26.9)$ & $20(40)$ & 1.589 & 0.173 & $0.82-3.09$ \\
\hline Grade 2 & $1(3.8)$ & $4(8)$ & 2.252 & 0.156 & $0.74-6.90$ \\
\hline Grade 3 & 0 & $7(14)$ & 2.952 & 0.020 & $1.19-7.32$ \\
\hline \multicolumn{6}{|l|}{ Shock } \\
\hline Absent (ref) & $13(50)$ & $26(52)$ & - & - & - \\
\hline Present & $13(50)$ & $24(48)$ & 1.22 & 0.49 & $0.69-2.16$ \\
\hline \multicolumn{6}{|l|}{ CNS complications } \\
\hline Absent (ref) & $26(100)$ & $46(92)$ & - & - & - \\
\hline Present & 0 & $4(8)$ & 1.565 & 0.390 & $0.56-4.34$ \\
\hline \multicolumn{6}{|l|}{ Thromboembolic complications } \\
\hline Absent (ref) & $24(92.3)$ & $46(92)$ & - & - & - \\
\hline Present & $2(7.7)$ & $4(8)$ & 1.565 & 0.390 & $0.56-4.34$ \\
\hline \multicolumn{6}{|l|}{ Severity-median (IQR) } \\
\hline SOFA score on admission ${ }^{*}$ & $3(3-4)$ & $4(3-7)$ & 1.258 & $<0.001$ & $1.15-1.38$ \\
\hline SOFA score on last day in ICU* & $2(2-3)$ & $8(6-11)$ & 1.162 & 0.001 & $1.07-1.27$ \\
\hline
\end{tabular}


Table 3: (Contd...)

\begin{tabular}{|c|c|c|c|c|c|}
\hline & Survived $(n=26)$ & $\operatorname{Died}(n=50)$ & Hazard ratio & $p$ value & $95 \% \mathrm{Cl}$ \\
\hline \multicolumn{6}{|l|}{ Treatment } \\
\hline \multicolumn{6}{|l|}{ Antivirals } \\
\hline No (ref) & $16(61.5)$ & $33(66)$ & - & - & - \\
\hline Yes & $10(38.5)$ & $17(34)$ & 0.755 & 0.365 & $0.41-1.38$ \\
\hline \multicolumn{6}{|l|}{ Need of vasopressors $n(\%)^{*}$} \\
\hline No (ref) & $23(92)$ & $4(8)$ & - & - & - \\
\hline Yes & $2(8)$ & $46(92)$ & 0.362 & 0.068 & $0.12-1.08$ \\
\hline \multicolumn{6}{|c|}{ Oxygen supplementation and mechanical ventilation } \\
\hline $\begin{array}{l}\text { Median (IQR) days of oxygen } \\
\text { supplementation }\end{array}$ & $8(5-15)$ & $7(5-15)$ & 0.773 & $<0.001$ & $0.71-0.841$ \\
\hline \multicolumn{6}{|l|}{ Noninvasive ventilation } \\
\hline No (ref) & $3(11.5)$ & $1(2)$ & - & - & - \\
\hline Yes & $23(88.5)$ & $49(98)$ & 1.631 & 0.630 & $0.22-11.94$ \\
\hline $\begin{array}{l}\text { Median (IQR) days of } \\
\text { noninvasive ventilation }\end{array}$ & $3(3-7)$ & $3(1-6)$ & 0.808 & $<0.001$ & $0.73-0.90$ \\
\hline $\begin{array}{l}\text { Invasive mechanical } \\
\text { ventilation duration }\end{array}$ & $6(4-10)$ & $6(3-22)$ & $6(4-8)$ & 0.62 & $0.92-1.98$ \\
\hline \multicolumn{6}{|l|}{ Invasive line ${ }^{*}$} \\
\hline No (ref) & $18(72)$ & $6(12)$ & - & - & - \\
\hline Yes & $7(28)$ & $44(88)$ & 0.679 & 0.383 & $0.28-1.62$ \\
\hline \multicolumn{6}{|l|}{ Diuretics $^{*}$} \\
\hline No (ref) & $20(76.9)$ & $32(64)$ & - & - & - \\
\hline Yes & $5(19.2)$ & $18(36)$ & 0.750 & 0.337 & $0.42-1.35$ \\
\hline \multicolumn{6}{|l|}{ Renal replacement therapy ${ }^{*}$} \\
\hline No (ref) & $25(100)$ & $42(84)$ & - & - & - \\
\hline Yes & 0 & $8(16)$ & 1.639 & 0.209 & $0.76-3.54$ \\
\hline \multicolumn{6}{|l|}{ Tissue plasminogen activator } \\
\hline No (ref) & $22(84.6)$ & $45(90)$ & - & - & - \\
\hline Yes & $4(15.4)$ & $5(10)$ & 1.771 & 0.238 & $0.69-4.57$ \\
\hline \multicolumn{6}{|l|}{ Need of transfusion $n(\%)$} \\
\hline No (ref) & $24(92.3)$ & $36(72)$ & - & - & - \\
\hline Yes & $2(7.7)$ & $14(28)$ & 1.276 & 0.453 & $0.68-2.41$ \\
\hline \multicolumn{6}{|l|}{ Surgery } \\
\hline No (ref) & $26(100)$ & $47(94)$ & - & - & - \\
\hline Yes & 0 & $3(6)$ & 1.711 & 0.375 & $0.523-5.60$ \\
\hline \multicolumn{6}{|l|}{ Outcome } \\
\hline Total ICU stay & $9(6-16)$ & $8(5-15)$ & 0.235 & $<0.001$ & $0.15-0.367$ \\
\hline Total hospital stay & $15(10-20)$ & $10.5(6-17)$ & 0.682 & $<0.001$ & $0.60-0.776$ \\
\hline
\end{tabular}

${ }^{*} 1$ data missing; ${ }^{* *} 2$ data missing; ${ }^{* * *} 5$ data missing; \#8 data missing

Europe and Asia. ${ }^{7}$ Further, non-Chinese patients have a higher frequency of diarrhea, nausea/vomiting, and abdominal pain as compared to Chinese patients. ${ }^{8}$ Cultural differences, dietary habits, innate immunity, and various other factors may influence clinical presentation and extrapolating data from Europe, America, Australia, or China may not be appropriate in the Indian scenario.
Jin et al. reported gastrointestinal symptoms in $22.97 \%$ of patients with severe COVID-19 illness. ${ }^{9}$ Ghoshal et al. observed that severe/critical disease was present in 13/44 patients with digestive symptoms. ${ }^{6}$ In our study, both digestive and nondigestive symptoms were present in $30.34 \%$ of patients. Frequency of digestive symptoms in our study was higher as compared to the literature from Asia. ${ }^{7}$ This may be attributed to increased 


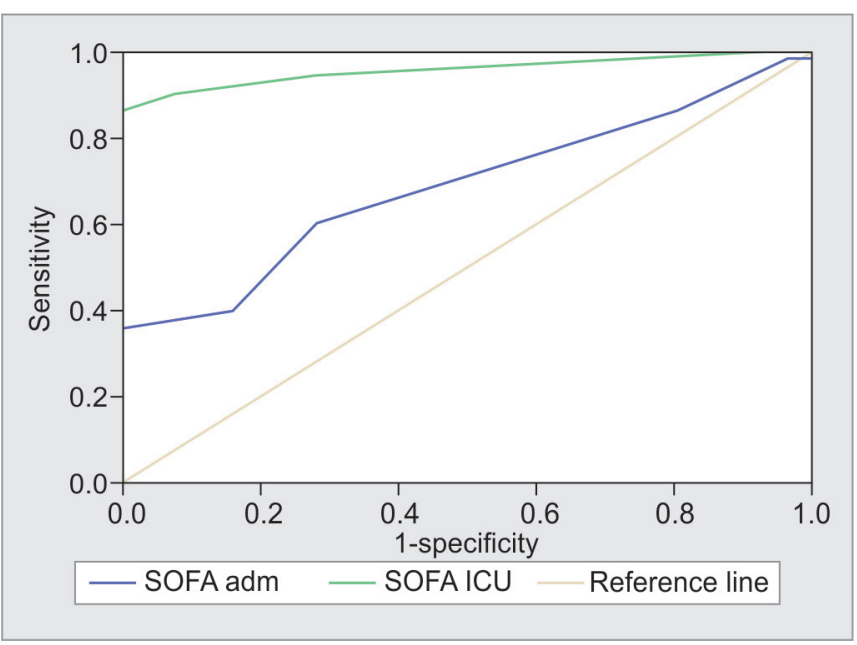

Fig. 2: ROC curve analysis

Table 4: Receiver-operating curve of SOFA score as a predictor of mortality

\begin{tabular}{lccc}
\hline & $\begin{array}{c}\text { Area under } \\
\text { the curve }\end{array}$ & $p$ value & $\begin{array}{c}95 \% \text { confidence } \\
\text { interval }\end{array}$ \\
\hline SOFA on admission & 0.685 & 0.010 & $0.57-0.80$ \\
SOFA on last day ICU & 0.954 & 0.000 & $0.91-1.00$ \\
\hline
\end{tabular}

awareness of nonrespiratory symptoms and not asking specific history of each digestive symptom. Further, we studied only severe COVID-19 patients, where frequency of digestive symptoms may be higher. High density and virulence of the virus may damage the digestive system. ${ }^{3}$ As the severity of infection with severe acute respiratory syndrome coronavirus 2 (SARS-CoV-2) increases, the digestive symptoms are reported to be more pronounced. ${ }^{10}$ Digestive symptoms indicate viral load and replication within the gastrointestinal tract, increasing disease severity. ${ }^{3}$

Pan et al. reported the loss of appetite, diarrhea, vomiting, and abdominal pain in severe illness with frequency of 100, 21.4, 7.14, and $0 \%$, respectively with higher abdominal pain in critical patients $(8.70 \%){ }^{3}$ We noted a similar frequency of loss of appetite (100\%), less frequent diarrhea (7.8\%) though higher frequency of nausea (75\%), vomiting (32.89\%), abdominal distension (26.3\%), abdominal pain $(13.15 \%)$, and gastrointestinal bleeding (7.8\%) was present. Abdominal pain is associated with increased severity of COVID-19 illness. ${ }^{11}$ In two patients with nausea, vomiting, loss of appetite, and acute abdominal pain, acute abdomen was diagnosed necessitating emergency laparotomy.

Male gender, low BMI, and low fibrinogen were observed to be predictors of digestive symptoms. Low fibrinogen and low platelet count may reflect a coagulation disorder in severe COVID-19, which can lead to microthrombosis in different organ systems. Loss of intestinal barrier integrity and gut microbes can activate the release of proinflammatory cytokines, leading to systemic inflammation. ${ }^{12}$ Gastrointestinal symptoms have previously been associated with younger age and multiple comorbidities. ${ }^{13,14}$

We did not study the mechanistic pathways for involvement of the digestive system in COVID-19. With the presence of both respiratory and digestive symptoms in 71 patients, the vital crosstalk between lung and gut cannot be overemphasized. Lung-gut axis is bidirectional as microbial metabolites and endotoxins can modulate the lung through blood and lung inflammation can in turn alter the gut microbiota. ${ }^{3}$ Angiotensin-converting enzyme-2 (ACE-2) receptors in endothelium and smooth muscle cells of the colon and surface cells of small intestine may serve as an entry pathway for direct cytopathic attack. ${ }^{15}$ Further, hypoxemia may exacerbate gastrointestinal mucosal injury, leading to ulceration and bleeding. ${ }^{1}$ Secondary damage due to inflammation and hypercoagulation state may further increase cell injury and endotoxemia, causing poor outcomes. ${ }^{16}$ New onset symptoms of gastrointestinal tract heralding severe COVID-19 can be part of systemic hyperinflammation seen during the second phase of COVID-19 illness. ${ }^{17}$

Frequency of liver injury at admission was $30.8 \%$ similar to that reported previously (7.6-39\%). ${ }^{15}$ Mild liver injury was most frequent (26.5\%). The liver may be targeted in COVID-19 by the immune system activation, triggering of a cytokine, besides direct viral attack through portal circulation and drug-induced hepatotoxicity. ${ }^{18-21}$

\section{Impact of Digestive Symptoms on Course of Hospital Stay}

Shorter time from symptom onset to hospital admission (5 vs 4 days) in our study as compared to published literature in COVID-19 (9.0 vs 7.3 days) may be due to increased awareness and subsequent earlier admission. ${ }^{3}$

In our study, COVID-19 patients with digestive symptoms did not have severe inflammation (D-dimers, NLR) at admission. Similar lower inflammatory biomarkers in patients with gastrointestinal symptoms were present in a retrospective study from China and the United States of America., ${ }^{9,18}$ They hypothesized that lower degree of inflammation may modulate rates of adverse outcomes, but impaired immune response may lead to inability to clear the virus, resulting in prolonged duration of illness. The change in gut microbiota has been associated with changes in the immune system and susceptibility to suffer more severe clinical consequences of COVID-19. ${ }^{12}$ So, the digestive group needed more interventions with a higher need for blood transfusion, exploratory laparotomy, and noninvasive ventilation. The mean hemoglobin and albumin tended to decrease over time.

Higher incidence of acute renal insufficiency is reported in COVID-19 patients with gastrointestinal symptoms (9.3 vs $3.1 \%) .{ }^{22}$ The incidence of AKI, ALI, and ARDS in our digestive cohort was much higher as compared to published literature. ${ }^{3}$ This may be attributed to inclusion of only severe COVID-19 illness in our study. Heart failure was indeed higher, though no difference was found in the incidence of any other organ-specific complication as compared to the nondigestive group. Impaired intestinal functional barriers and increased inflammatory cytokine activation may promote cardiac involvement in COVID-19 patients. ${ }^{23}$ Endotoxemia, increased gut permeability, and systemic sepsis may have been higher in our digestive patients, though we did not study sepsisrelated parameters as they could have multiple contributory confounders.

Gastrointestinal symptoms have been associated with a longer indolent illness with lower mortality (0.0 vs $5 \%) .{ }^{5,14}$ In contrast to these, we observed a significantly higher mortality in patients with digestive symptoms, though time-based parameters remained similar between the groups. So, while initial inflammatory markers were not significantly raised in the digestive group, course of illness was turbulent with higher proportional mortality rate. 


\section{Predictors of Mortality in Digestive Group}

In past studies in patients admitted with COVID-19, diarrhea, liver injury at presentation, older age, number of comorbidities, tachypnea, and severe hypoxia have been identified as independent predictors of mortality. ${ }^{7,24,25}$

We observed a higher risk (aHR 4.3, $p<0.02$ ) of mortality in patients with digestive symptoms with chronic neurological disorders than patients presenting with only chronic neurological disease (HR 2.129). ${ }^{26}$ The presence of multiple digestive symptoms may support the presence of both upper and lower gastrointestinal inflammation with activation of a sepsis-like state. ${ }^{27}$ We hypothesize that compromised nutritional status, decreased immunity, and predisposition to infection may be responsible for mortality in patients with both digestive symptoms and neurological disorders. Furthermore, patients with hepatobiliary diseases and chronic liver disease are already in a precarious balance, which is easily decompensated in the presence of COVID-19 (aHR 2.829, $p$ 0.05). The presence of severe $\mathrm{AKI}$ as a predictor of mortality has been seen in earlier studies in COVID-19 patients also. ${ }^{28}$

SOFA score is often used for prognostication in critical care patients. In a study exploring effectiveness of SOFA score, a value of 3 was used to predict inhospital mortality in critically ill COVID-19 patients with a sensitivity and specificity of 90.00 and $83.18 \%$, respectively. ${ }^{29}$ We observed that in nonsurvivors with preadmission digestive symptoms, a SOFA score of 5.5 at admission and 4.5 at discharge was present with a sensitivity of 36 and $86 \%$ and specificity of $100 \%$, respectively Table 4 .

We found that as the duration of noninvasive or invasive ventilation and ICU or hospital stay increased, there was less association with mortality. This resonates with earlier studies where patients who were discharged alive had longer length of stay than those who died..$^{30,31}$ It is not surprising, as patients who are critical may pass away soon after hospital/ICU admission, automatically leading to a decrease in duration of any intervention or hospital/ ICU stay.

As time progresses, patients within the digestive group may be more vulnerable to endotoxemia and subsequent sepsis with the need for vasopressors to maintain perfusion. After adjusting for all confounders, vasopressor use was associated with 11.58 times higher risk of mortality. It is prudent to identify predictors of mortality at admission and during illness for triage, timely intervention, prognostication, and optimum resource allocation in patients with preadmission digestive symptoms with severe COVID-19.

\section{Strength of Our Study}

- Our data collection was prospective with specific history of digestive symptoms taken at admission, hence ensuring that no patient was missed.

- We included preadmission digestive symptoms only to negate the influence of polypharmacy, change in diet, and nosocomial infection on the gastrointestinal system.

- Team which collected data during the course of hospital stay and final outcome was blinded to the group allocation of the patient thus removing investigator bias.

- We have holistically studied the clinical parameters, laboratory investigations, organ-specific complications, and invasive interventions in medical course in each patient with and without digestive symptoms in COVID-19.
- We have summarized risk factors of digestive symptoms in severe COVID-19 as well as predictors of mortality found in these patients, which may help in better prognostication, optimization, and resource allocation.

\section{Limitations}

- It is a single-center study which may decrease the external validation of our results. However, our institute is the main referral center for patients from Madhya Pradesh and neighboring states. Hence, the results may be generalized to central India till there is availability of more data.

- Since we studied only severe COVID-19illness needing intensive care, organ-specific complications and overall mortality were more than reported previously.

- We did not study interleukin-6 and serial D-dimers to study the cytokine response due to institutional policy. However, we used NLR, CRP, and fibrinogen to study the same.

- Our study finding of higher incidence of heart failure in COVID-19 associated with digestive symptoms is confined by limitation of difficulty in removing confounders in observational studies of alcohol abuse, smoking, and sedentary lifestyle, though we did assess for age, gender distribution, BMI, comorbidities like hypertension, preexisting coronary artery disease, and diabetes between the survivors and nonsurvivors in the digestive group.

\section{Conclusion}

This study demonstrates that patients with preadmission digestive symptoms in severe COVID-19 may have less inflammation at admission, but need more invasive interventions with higher incidence of heart failure and proportional mortality rate. Possibility of confounding factors for heart failure exists.

\section{Acknowledgments}

The authors acknowledge the work done by all residents and nursing staff tirelessly during the pandemic in taking care of COVID-19 patients admitted to our intensive care unit.

\section{ORCID}

Sunaina Tejpal Karna @ https://orcid.org/0000-0002-4200-5948

Pooja Singh (1) https://orcid.org/0000-0003-0556-7359

Gouroumourty Revadi i https://orcid.org/0000-0002-9646-4971

Alkesh Khurana (1) https://orcid.org/0000-0002-9646-4971

Aishwary Shivhare 이 https://orcid.org/0000-0001-5896-5612

Saurabh Saigal @ https://orcid.org/0000-0002-2364-2271

ManojKumar Rathiswamy @ https://orcid.org/0000-0002-4990-3708

Jai Prakash Sharma 1 https://orcid.org/0000-0003-4147-1637

Vaishali Waindeskar (1) https://orcid.org/0000-0002-0556-7159

\section{References}

1. Tian $Y$, Rong L, Nian W, He Y. Review article: gastrointestinal features in COVID-19 and the possibility of faecal transmission. Aliment Pharmacol Ther 2020;51(9):843-851. DOI: 10.1111/apt.15731.

2. Portes SA, Volotão Ede M, Rocha MS, Rebelo MC, Xavier MD, Assis $\mathrm{RM}$, et al. A non-enteric adenovirus A12 gastroenteritis outbreak in Rio de Janeiro, Brazil. Mem Inst Oswaldo Cruz 2016;111(6):403-406. DOI: 10.1590/0074-02760160030.

3. Pan L, Mu M, Yang P, Sun Y, Wang R, Yan J, et al. Clinical characteristics of COVID-19 patients with digestive symptoms in Hubei, China: a 
descriptive, cross-sectional, multicenter study. Am J Gastroenterol 2020;115(5):766-773. DOI: 10.14309/ajg.0000000000000620.

4. Luo S, Zhang X, Xu H. Don't overlook digestive symptoms in patients with 2019 novel coronavirus disease (COVID-19). Clin Gastroenterol Hepatol 2020;18(7):1636-1637. DOI: 10.1016/j.cgh.2020.03.043.

5. Nobel YR, Phipps M, Zucker J, Lebwohl B, Wang TC, Sobieszczyk $\mathrm{ME}$, et al. Gastrointestinal symptoms and coronavirus disease 2019: a case-control study from the United States. Gastroenterology 2020;159(1):373-375.e2. DOI: 10.1053/j.gastro.2020.04.017.

6. Ghoshal UC, Ghoshal U, Mathur A, Singh RK, Nath A, Garg A, et al. The spectrum of gastrointestinal symptoms in patients with coronavirus disease-19: predictors, relationship with disease severity, and outcome. Clin Transl Gastroenterol 2020;11(12):e00259. DOI: 10.14309/ ctg.0000000000000259.

7. Aziz M, Haghbin H, Lee-Smith W, Goyal H, Nawras A, Adler DG. Gastrointestinal predictors of severe COVID-19: systematic review and meta-analysis. Ann Gastroenterol 2020;33(6):615-630. DOI: 10.20524/ aog.2020.0527.

8. Akin H, Kurt R, Tufan F, Swi A, Ozaras R, Tahan V, et al. Newly reported studies on the increase in gastrointestinal symptom prevalence with COVID-19 infection: a comprehensive systematic review and metaanalysis. Diseases 2020;8(4):41. DOI: 10.3390/diseases8040041.

9. Jin X, Lian JS, Hu JH, Gao J, Zheng L, Zhang YM, et al. Epidemiological, clinical and virological characteristics of 74 cases of coronavirusinfected disease 2019 (COVID-19) with gastrointestinal symptoms. Gut 2020;69(6):1002-1009. DOI: 10.1136/gutjnl-2020-320926.

10. Hunt RH, East JE, Lanas A, Malfertheiner P, Satsangi J, Scarpignato C, et al. COVID-19 and gastrointestinal disease: implications for the gastroenterologist. Dig Dis 2021;39(2):119-139. DOI: 10.1159/000512152.

11. Sonkar C, Kashyap D, Varshney N, Baral B, Jha HC. Impact of gastrointestinal symptoms in COVID-19: a molecular approach. SN Compr Clin Med 2020;1-12. DOI: 10.1007/s42399-020-00619-z.

12. Villapol S. Gastrointestinal symptoms associated with COVID-19: impact on the gut microbiome. Transl Res 2020;226:57-69. DOI: 10.1016/j.trsl.2020.08.004.

13. Eslick GD. Gastrointestinal symptoms and obesity: a meta-analysis. Obes Rev 2012;13(5):469-479. DOI: 10.1111/j.1467-789X.2011.00969.x.

14. Laszkowska M, Faye AS, Judith, Truong H, Silver ER, Ingram M, et al. Disease course and outcomes of COVID-19 among hospitalized patients with gastrointestinal manifestations. Clin Gastroenterol Hepatol 2020;S1542-3565(20)31367-7. DOI: 10.1016/j.cgh.2020.09.037.

15. Zhong $P$, Xu J, Yang D, Shen Y, Wang L, Feng Y, et al. COVID-19associated gastrointestinal and liver injury: clinical features and potential mechanisms. Signal Transduct Target Ther 2020;5(1):256. DOI: 10.1038/s41392-020-00373-7.

16. Ignat M, Philouze G, Aussenac-Belle L, Faucher V, Collange $O$, Mutter D, et al. Small bowel ischemia and SARS-CoV-2 infection: an underdiagnosed distinct clinical entity. Surgery 2020;168(1):14-16. DOI: 10.1016/j.surg.2020.04.035.

17. Mao R, Qiu Y, He JS, Tan JY, Li XH, Liang J, et al. Manifestations and prognosis of gastrointestinal and liver involvement in patients with COVID-19: a systematic review and meta-analysis. Lancet Gastroenterol Hepatol 2020;5(7):667-678. DOI: 10.1016/S24681253(20)30126-6.
18. Hayden JA, van der Windt DA, Cartwright JL, Côté P, Bombardier C. Assessing bias in studies of prognostic factors. Ann Intern Med 2013;158(4):280-286. DOI: 10.7326/0003-4819-158-4-201302190-00009.

19. Aziz M, Fatima R, Assaly R. Elevated interleukin- 6 and severe COVID-19: a meta-analysis. J Med Virol 2020;92(11):2283-2285. DOI: 10.1002/jmv.25948.

20. Aziz M, Fatima R, Lee-Smith W, Assaly R. The association of low serum albumin level with severe COVID-19: a systematic review and metaanalysis. Crit Care 2020;24(1):255. DOI: 10.1186/s13054-020-02995-3.

21. Zippi M, Fiorino S, Occhigrossi G, Hong W. Hypertransaminasemia in the course of infection with SARS-CoV-2: incidence and pathogenetic hypothesis. World J Clin Cases 2020;8(8):1385-1390. DOI: 10.12998/ wjcc.v8.i8.1385.

22. Cholankeril G, Podboy A, Aivaliotis VI, Pham EA, Spencer SP, Kim $D$, et al. Association of digestive symptoms and hospitalization in patients with SARS-CoV-2 infection. Am J Gastroenterol 2020;115(7):1129-1132. DOI: 10.14309/ajg.0000000000000712.

23. Hoel H, Heggelund L, Reikvam DH, Stiksrud B, Ueland T, Michelsen $A E$, et al. Elevated markers of gut leakage and inflammasome activation in COVID-19 patients with cardiac involvement. J Intern Med 2021;289(4):523-531. DOI: 10.1111/joim.13178.

24. Kumar A, Arora A, Sharma P, Anikhindi SA, Bansal N, Singla V, et al. Gastrointestinal and hepatic manifestations of Corona Virus Disease19 and their relationship to severe clinical course: a systematic review and meta-analysis. Indian J Gastroenterol 2020;39(3):268-284. DOI: 10.1007/s12664-020-01058-3.

25. Hajifathalian K, Krisko T, Mehta A, Kumar S, Schwartz R, Fortune $B$, et al. Gastrointestinal and hepatic manifestations of 2019 novel coronavirus disease in a large cohort of infected patients from New York: clinical implications. Gastroenterology 2020;159(3):1137-1140. e2. DOI: 10.1053/j.gastro.2020.05.010.

26. García-Azorín D, Martínez-Pías E, Trigo J, Hernández-Pérez I, VallePeñacoba G, Talavera B, et al. Neurological comorbidity is a predictor of death in Covid-19 disease: a cohort study on 576 patients. Front Neurol 2020;11:781. DOI: 10.3389/fneur.2020.00781.

27. Jiang Y, Han C, Bai T, Zhang S, Song J, Hou X. Gastroenterologists reveal more digestive symptoms in COVID-19 patients than nongastroenterologists in fever clinic. Gastroenterol Res Pract 2020;2020:8853922. DOI: 10.1155/2020/8853922.

28. Chen N, Zhou M, Dong X, Qu J, Gong F, Han Y, et al. Epidemiological and clinical characteristics of 99 cases of 2019 novel coronavirus pneumonia in Wuhan, China: a descriptive study. Lancet 2020;395(10223):507-513. DOI: 10.1016/S0140-6736(20)30211-7.

29. Liu S, Yao N, Qiu Y, He C. Predictive performance of SOFA and qSOFA for in-hospital mortality in severe novel coronavirus disease. Am J Emerg Med 2020;38(10):2074-2080. DOI: 10.1016/j.ajem.2020.07.019.

30. Richardson S, Hirsch JS, Narasimhan M, Crawford JM, McGinn T, Davidson KW, et al. Presenting characteristics, comorbidities, and outcomes among 5700 patients hospitalized with COVID-19 in the New York City area [published correction appears in JAMA 2020;323(20):2098]. JAMA 2020;323(20):2052-2059. DOI: 10.1001/ jama.2020.6775.

31. Rees EM, Nightingale ES, Jafari Y, Waterlow NR, Clifford S, Pearson CA, et al. COVID-19 length of hospital stay: a systematic review and data synthesis. BMC Med 2020;18(1):270. DOI: 10.1186/s12916-020-01726-3. 